\title{
Impact of the additive ketamine or fentanyl on quality of sedation in endoscopic ultrasonography with propofol
}

\author{
Daskaya H., Uysal H., Yılmaz İnal F., Esen A., Karaarslan K \\ Bezmialem Foundation University, Faculty of Medicine, Department of Anesthesiology, Istanbul, Turkey
}

\section{Background and Goal of Study:}

Application of the endoscopic procedures under anesthesia was increased according to improve patients comfort and satisfaction.

Various medical combinations are used for this manner. Here in this study, we aimed to compare the quality of sedation on endoscopic ultrasonography (EUS) application with propofol- fentanyl and propofolketamin combination.

\section{Materials and methods:}

Ninety patients with scheduled EUS were enrolled to study. After

premedication, sedation anesthesia was performed with 1,5 mg.kg-1 Propofol, $1 \mathrm{mg} . \mathrm{kg}-1$ Ketamin in Group $\mathrm{K}$ and 1,5 mg.kg-1 Propofol, 1 mcg.kg1 Fentanil in Grup F. In the situation of inadequate anesthesia, additive 0,2 $\mathrm{mg} / \mathrm{kg}$ Propofol was performed.

Richmond Agitation Score (RAS), hear rate (HR), mean systolic-diastolic blood pressure, peripheral oxygen saturation (SpO2), duration of procedure and used propofol doses were recorded. Nausea and vomiting, Numeric Rank Score, visual analog scale (VAS) and Aldrete score were recorded postoperatively. The satisfaction of doctor and patients were scored between 0-4.

\section{Results and discussion:}

Total used propofol dose and PACU discharge time were significantly lower in Group $F(p<0.001)$. Intraoperative $\mathrm{HR}$ and TA were more stable in Group F rather than Group K (p:0.002) however intraoperative SpO2 levels were similar between groups. HR, TA at the entrance of PACU and PACU discharge time was significantly increased in Group K ( $<<0.001)$. Patients satisfaction was significantly improved in Group F (p:0.01) but no difference was found for doctor's satisfaction.

Conclusion(s): Propofol- Fentanyl combination is found to be more comfortable in esophageal, gastric and pancreaticobilier EUS application especially in advanced age and the presence of concomitant disease rather than propofol- ketamin combination.

\section{References: \\ 1. John K Triantafillidis, Emmanuel Merikas, Dimitrios Nikolakis, Apostolos E Papalois. Sedation in gastrointestinal endoscopy: Current issues. World $\mathrm{J}$ Gastroenterol 2013 January 28; 19(4): 463-481 2. Saurabh Sethi, Vaibhav Wadhwa, Adarsh Thaker et. all. Propofol versus traditional sedative agents for advanced endoscopic procedures: A meta-analysis. Digestive Endoscopy 2013; doi: 10.1111/den.12219 Ethical Research Declaration: \\ 1. Prospective observational only studies Preferred Presentation Type: Clinical or experimental study.}

